\title{
Almost L-Dunford-Pettis sets in Banach lattices and its applications
}

\author{
Abderrahman Retbi (C) \\ Ibn Tofail University, Faculty of Sciences, Department of Mathematics, B.P. 133, Kenitra, Morocco
}

\begin{abstract}
We introduce and study the notion of almost L-Dunford-Pettis sets in Banach lattices and we give some characterizations of it in terms of sequences. As an application, we establish new properties of almost Dunford-Pettis completely continuous operators. Finally, by introducing the concept of aL-Dunford-Pettis property in Banach lattices, we investigate the weak compactness of almost Dunford-Pettis completely continuous operator.
\end{abstract}

Mathematics Subject Classification (2010). 46A40, 46B 40

Keywords. Banach lattice, Dunford-Pettis set, relatively compact Dunford-Pettis property, Dunford-Pettis completely continuous operator

\section{Introduction and notation}

A norm bounded subset $A$ of a Banach space $X$ is said to be Dunford-Pettis set, if every weakly null sequence $\left(f_{n}\right)$ in $X^{\prime}$ converges uniformly to zero on $\mathrm{A}$, that is, $\lim _{n \rightarrow \infty} \sup _{x \in A} f_{n}(x)=0$. Recall from [6] that a norm bounded subset $A$ of a topological dual Banach space $X^{\prime}$ is an L-Dunford-Pettis if every weakly null sequence $\left(x_{n}\right)$, which is a Dunford-Pettis subset of $X$ converges uniformly to zero on A, that is $\lim _{n \rightarrow \infty} \sup _{f \in A} f\left(x_{n}\right)=$ 0 .

A Banach space $X$ has

- the relatively compact Dunford-Pettis property (DPrcP for short) if every weakly null sequence, which is a Dunford-Pettis set in $X$, is norm null [7].

- the L-Dunford-Pettis property if every L-Dunford-Pettis set in $X^{\prime}$ is relatively weakly compact [6].

A Banach lattice $E$ has the positive relatively compact Dunford-Pettis property (PDPrcP for short) if every disjoint weakly null sequence, which is a Dunford-Pettis set in $X$, is norm null [4]. Note that if a Banach lattice $E$ has the DPrcP then, it has PDPrcP but the converse is not true in general (see Example 3.4 of [4]).

An operator $T$ from a Banach space $X$ into a another Banach space $Y$ is called DunfordPettis completely continuous (DPcc for short) if each weakly null sequence $\left(x_{n}\right)$, which is a Dunford-Pettis set in $X$, we have $\left\|T\left(x_{n}\right)\right\|_{Y} \rightarrow 0$, as $n \rightarrow \infty$ [7]. Recall from [4] that an operator $T$ from a Banach lattice $E$ into a Banach space $Y$ is called almost Dunford-Pettis completely continuous (aDPcc for short) if each disjoint weakly null sequence $\left(x_{n}\right)$, which is a Dunford-Pettis set in $E$, we have $\left\|T\left(x_{n}\right)\right\|_{Y} \rightarrow 0$, as $n \rightarrow \infty$.

Email address: abderrahmanretbi@hotmail.com

Received: 23.12.2016; Accepted: 30.01.2019 
Dunford-Pettis sets definition is given firstly by K.T. Andrews [2] as a norm bounded subset $A$ of a Banach space $X$ is a Dunford-Pettis set whenever every weakly compact operator from $X$ to an arbitrary Banach space $Y$ carries $A$ to a norm totally bounded set. Then Andrew characterized the Dunford-Pettis sets by using sequences $\left(f_{n}\right)$ in $X^{\prime}$. Recently in [3], Bouras considered the disjoint version of the Dunford-Pettis sets and introduced the almost Dunford-Pettis sets in Banach lattices. Following Bouras, a bounded subset $A$ of a Banach lattice $E$ is said to be an almost Dunford-Pettis set if every disjoint weakly null sequence $\left(f_{n}\right)$ in $E^{\prime}$ converges uniformly to zero on A. In this paper, using the disjoint sequence techniques we consider the disjoint version of L-Dunford-Pettis sets, that we call almost L-Dunford-Pettis sets in Banach lattices (Definition 2.1). In addition, we introduce the aL-Dunford-Pettis property which is shared by those Banach lattice whose every almost L-Dunford-Pettis subset of his topological dual is relatively weakly compact (Definition 4.1).

The article is organized as follows. In Section 2 we establish some characterizations of almost L-Dunford-Pettis set in terms of sequences (Proposition 2.2), and we show that each order interval in a dual Banach lattice is an almost L-Dunford-Pettis set (Proposition 2.4). Also, we give some equivalent condition for $T^{\prime}(A)$ to be almost L-Dunford-Pettis set where $A$ is a norm bounded solid subset of $E$ and $T: E \rightarrow F$ is an order bounded operator between two Banach lattices (Theorem 2.7). In Section 3, using the notion of almost L-Dunford-Pettis set, we give characterizations of aDPcc operator and PDPrcP (Theorem 3.1 and Corollary 3.2). After that, we characterize Banach lattice $E$ such that each almost L-Dunford-Pettis set of $E^{\prime}$ is L-Dunford-Pettis (Theorem 3.12), and we derive some sufficient conditions such that the PDPrcP coincide with the DPrcP (Corollary 3.13). In Section 4, we prove that a Banach lattice $E$ has the aL-Dunford-Pettis property if and only if each aDPcc operator from a Banach lattice $E$ into any Banach space $Y$ is weakly compact (Theorem 4.2), and we deduce an important result about the reflexive space (Corollary 4.3).

To state our results, we need to fix some notations and recall some definitions. A Banach lattice is a Banach space $(E,\|\cdot\|)$ such that $E$ is a vector lattice and its norm satisfies the following property: for each $x, y \in E$ such that $|x| \leq|y|$, we have $\|x\| \leq\|y\|$. If $E$ is a Banach lattice, its topological dual $E^{\prime}$, endowed with the dual norm, is also a Banach lattice. The sequence $\left(x_{n}\right)$ of a Banach lattice $E$ is disjoint if $\left|x_{n}\right| \wedge\left|x_{m}\right|=0, n \neq m$ (we denote by $\left.x_{n} \perp x_{m}\right)$.

Recall that a nonzero element $x$ of a vector lattice $G$ is discrete if the order ideal generated by $x$ equals the subspace generated by $x$. The vector lattice $G$ is discrete, if it admits a complete disjoint system of discrete elements. The lattice operations of a Banach lattice $E$ are weakly sequentially continuous, whenever $x_{n} \rightarrow 0$ for $\sigma\left(E, E^{\prime}\right)$ as $n \rightarrow \infty$ imply $\left|x_{n}\right| \rightarrow 0$ for $\sigma\left(E, E^{\prime}\right)$, as $n \rightarrow \infty$. We will use the term operator $T: X \longrightarrow Y$ between two Banach space to mean a bounded linear mapping, its dual operator $T^{\prime}$ is defined from $Y^{\prime}$ into $X^{\prime}$ by $T^{\prime}(f)(x)=f(T(x))$ for each $f \in Y^{\prime}$ and for each $x \in X$. We refer the reader to [1] for unexplained terminology of Banach lattice theory and operators.

\section{Almost L-Dunford-Pettis set in a topological dual of Banach lattice}

We start this work by a definition of almost L-Dunford-Pettis set, which is a disjoint version of L-Dunford-Pettis set.

Definition 2.1. Let $E$ be a Banach lattice. A norm bounded subset $A$ of $E^{\prime}$ is called an almost L-Dunford-Pettis set, if every disjoint weakly null sequence $\left(x_{n}\right)$, which is a DP set in $E$ converge uniformly to zero on $A$, that is, $\lim _{n \rightarrow \infty} \sup _{f \in A}\left|f\left(x_{n}\right)\right|=0$.

Now, for a norm bounded subset of a topological dual Banach lattice, we give a characterization of an almost L-Dunford-Pettis sets. 
Proposition 2.2. Let $E$ be a Banach lattice and let $A$ be a norm bounded subset of $E^{\prime}$. The following statements are equivalent:

(1) $A$ is an almost L-Dunford-Pettis set in $E^{\prime}$.

(2) For every sequence $\left(f_{n}\right)$ in $A$ and every disjoint weakly null sequence $\left(x_{n}\right)$, which is a Dunford-Pettis set in $E$, we have $f_{n}\left(x_{n}\right) \rightarrow 0$ as $n \rightarrow \infty$.

Proof. $(2) \Rightarrow(1)$ Assume by way of contradiction that $A$ is not an almost L-DunfordPettis set in $E^{\prime}$. Then, there exists a disjoint weakly null sequence $\left(x_{n}\right)$, which is a Dunford-Pettis subset of $E$ such that $\sup _{f \in A}\left|f\left(x_{n}\right)\right|>\epsilon>0$ for some $\epsilon>0$ and each $n$. Hence, for every $n$ there exists some $f_{n}$ in $A$ such that $\left|f_{n}\left(x_{n}\right)\right|>\epsilon$, which is impossible from our hypothesis (2). This prove that $A$ is an almost L-Dunford-Pettis set in $E^{\prime}$.

$(1) \Rightarrow(2)$ Let $\left(f_{n}\right)$ be a sequence in $A$ and $\left(x_{n}\right)$ be a disjoint weakly null sequence, which is a Dunford-Pettis set in $E$. Since

$$
\left|f_{n}\left(x_{n}\right)\right| \leq \sup _{f \in A}\left|f\left(x_{n}\right)\right|,
$$

for every $n$, and $A$ is an almost L-Dunford-Pettis set in $E^{\prime}$ then, $f_{n}\left(x_{n}\right) \rightarrow 0$ as $n \rightarrow \infty$. This completes the proof.

As a consequence of Proposition 2.2, we obtain the following result.

Proposition 2.3. Let $E$ be a Banach lattice and let $\left(f_{n}\right)$ be a norm bounded sequence in $E^{\prime}$. The following statements are equivalent:

(1) The subset $\left\{f_{n}, n \in N\right\}$ is an almost L-Dunford-Pettis set in $E^{\prime}$.

(2) For every disjoint weakly null sequence $\left(x_{n}\right)$, which is a Dunford-Pettis set in E, we have $f_{n}\left(x_{n}\right) \rightarrow 0$ as $n \rightarrow \infty$.

The following proposition shows that every order interval in a topological dual Banach lattice is an almost L-Dunford-Pettis set.

Proposition 2.4. Let $E$ be a Banach lattice. Then, for every $f \in\left(E^{\prime}\right)^{+},[-f, f]$ is an almost L-Dunford-Pettis set in $E^{\prime}$.

Proof. Let $\left(x_{n}\right)$ be a disjoint weakly null sequence, which is a Dunford-Pettis set in $E$, and put $W=\left\{x_{n}: n \in N\right\}$. Then, $W$ is a relatively weakly compact set of $E$ and $\left(\left|x_{n}\right|\right)$ is a disjoint sequence in the solid hull of $W$. Now, by Theorem 4.34 of [1], we see that $\left(\left|x_{n}\right|\right)$ is a weakly null sequence of $E$. Since

$$
f\left(\left|x_{n}\right|\right)=\sup \left\{\left|g\left(x_{n}\right)\right|: g \in[-f, f]\right\} \rightarrow 0
$$

as $n \rightarrow \infty$ for all $f \in\left(E^{\prime}\right)^{+}$, it follows that $[-f, f]$ is an almost L-Dunford-Pettis set in $E^{\prime}$ for all $f \in\left(E^{\prime}\right)^{+}$, and this ends the proof.

From Proposition 2.4 and Theorem 1.73 of [1], we get

Corollary 2.5. Let $T$ be an order bounded operator from a Banach lattice $E$ into another Banach lattice $F$. Then, $T^{\prime}([-f, f])$ is an almost L-Dunford-Pettis set in $E^{\prime}$ for every $f \in\left(F^{\prime}\right)^{+}$.

Proof. Since $T$ be an order bounded operator from a Banach lattice $E$ into another Banach lattice $F$, by Theorem 1.73 of [1], we obtain that $T^{\prime}: F^{\prime} \rightarrow E^{\prime}$ is also order bounded. Thus, $T^{\prime}([-f, f])$ is an order bounded subset of $E^{\prime}$ for all $f \in\left(F^{\prime}\right)^{+}$, and so there exists $g \in\left(E^{\prime}\right)^{+}$such that $T^{\prime}([-f, f]) \subset[-g, g]$. Now, from Proposition 2.4, we conclude that $T^{\prime}([-f, f])$ is an almost L-Dunford-Pettis set in $E^{\prime}$ for every $f \in\left(F^{\prime}\right)^{+}$, as desired.

In order to prove the next theorem, we need the following lemma. 
Lemma 2.6. Let $E$ be a Banach lattice, and let $\left(g_{n}\right)$ be a norm bounded sequence in $E^{+}$. Then the sequence defined for $n \geq 2$ by

$$
f_{n}=\left(g_{n}-4^{n} \sum_{i=1}^{n-1} g_{i}-2^{-n} \sum_{i=1}^{\infty} 2^{-i} g_{i}\right)^{+},
$$

is a disjoint sequence of $E^{+}$.

Proof. Let $n>m \geq 2$, then

$$
0 \leq f_{n} \leq\left(g_{n}-4^{n} g_{m}\right)^{+},
$$

and

$$
\begin{aligned}
0 \leq 4^{n} f_{m} & \leq 4^{n}\left(g_{m}-4^{-n} g_{n}\right)^{+} \\
& =\left(4^{n} g_{m}-g_{n}\right)^{+} \\
& =\left(g_{n}-4^{n} g_{m}\right)^{-} .
\end{aligned}
$$

Since $\left(g_{n}-4^{n} g_{m}\right)^{+} \perp\left(g_{n}-4^{n} g_{m}\right)^{-}$, we deduce that $f_{n} \perp f_{m}$, as desired.

Theorem 2.7. Let $T$ be an order bounded operator from a Banach lattice $E$ into another Banach lattice $F$, and let $A$ be a norm bounded solid subset of $F^{\prime}$. The following statements are equivalent:

(1) $T^{\prime}(A)$ is an almost L-Dunford-Pettis set in $E^{\prime}$.

(2) $\left\{T^{\prime}\left(f_{n}\right), n \in N\right\}$ is an almost L-Dunford-Pettis set in $E^{\prime}$, for each disjoint sequence $\left(f_{n}\right) \subset A^{+}=A \cap\left(F^{\prime}\right)^{+}$.

Proof. (1) $\Rightarrow(2)$ Obvious.

$(2) \Rightarrow(1)$ Let $\left(x_{n}\right)$ be a disjoint weakly null sequence, which is a Dunford-Pettis set in $E$. To finish the proof, we have to prove that $\sup _{g \in A}\left|T^{\prime}(g)\left(x_{n}\right)\right| \rightarrow 0$ as $n \rightarrow \infty$. Assume by way of contradiction that $\sup _{g \in A}\left|T^{\prime}(g)\left(x_{n}\right)\right|$ does not converge to 0 as $n \rightarrow \infty$. So there exists some $\epsilon>0$ such that $\sup _{g \in A}\left|T^{\prime}(g)\left(x_{n}\right)\right|>\epsilon$ for each n. Hence, there exists $g_{n} \in A^{+}$such that $g_{n}\left(\left|T\left(x_{n}\right)\right|\right)>\epsilon$ for all natural number $n$. Let $g \in A^{+}$. Then from Corollary 2.5, we see that $T^{\prime}([g, g])$ is an almost L-Dunford-Pettis sets in $E^{\prime}$, and we have $g\left(\left|T\left(x_{n}\right)\right|\right) \rightarrow 0$ as $n \rightarrow \infty$. Let $n_{1}=1$. Since $g_{n_{1}}\left(T\left(x_{n}\right)\right) \rightarrow 0$ as $n \rightarrow \infty$, there exists some natural number $n_{2}$ such that $n_{2}>n_{1}=1$ and $g_{n_{1}}\left(\left|T\left(x_{n_{2}}\right)\right|\right)<\frac{\epsilon}{2^{2 \times 2+2}}$. Also, because $\sum_{k=1}^{2} g_{n_{k}}\left(\left|T\left(x_{n}\right)\right|\right) \rightarrow 0$ as $n \rightarrow \infty$, there exists some natural number $n_{3}$ such that $n_{3}>n_{2}>n_{1}=1$ and $\sum_{k=1}^{2} g_{n_{k}}\left(\left|T\left(x_{n_{3}}\right)\right|\right)<\frac{\epsilon}{2^{2 \times 3+2}}$. By induction, we get a strictly increasing subsequence $\left(n_{k}\right)$ of $N$ such that

$$
\left(\sum_{k=1}^{m-1} g_{n_{k}}\right)\left(\left|T\left(x_{n_{m}}\right)\right|\right)<\frac{\epsilon}{2^{2 m+2}} \text { for all } m \geq 2 .
$$

Now, let

$$
h=\sum_{k=1}^{\infty} 2^{-k} g_{n_{k}}
$$

and

$$
f_{m}=\left(g_{n_{m}}-4^{m} \sum_{k=1}^{m-1} g_{n_{k}}-2^{-m} h\right)^{+} \text {for all } m \geq 2 .
$$

So by Lemma 2.6, we see that $\left(f_{m}\right)$ is a disjoint sequence in $\left(F^{\prime}\right)^{+}$, as $0 \leq f_{m} \leq g_{n_{m}}$, $g_{n_{m}} \in A$ and $A$ is a solid subset of $F^{\prime}$ then, $f_{m} \in A^{+}$. Hence, we have

$$
\begin{aligned}
f_{m}\left(\left|T\left(x_{n_{m}}\right)\right|\right) & =\left(g_{n_{m}}-4^{m} \sum_{k=1}^{m-1} g_{n_{k}}-2^{-m} h\right)^{+}\left(\left|T\left(x_{n_{m}}\right)\right|\right) \\
& \geq\left(g_{n_{m}}-4^{m} \sum_{k=1}^{m-1} g_{n_{k}}-2^{-m} h\right)\left(\left|T\left(x_{n_{m}}\right)\right|\right) \\
& >\epsilon-\frac{\epsilon}{4}-2^{-m} h\left(\left|T\left(x_{n_{m}}\right)\right|\right) .
\end{aligned}
$$


This prove that $f_{m}\left(\left|T\left(x_{n_{m}}\right)\right|\right)>\frac{\epsilon}{2}$ for $m$ sufficiently large (because $2^{-m} h\left(\left|T\left(x_{n_{m}}\right)\right|\right) \rightarrow 0$ ). Since $f_{m}\left(\left|T\left(x_{n_{m}}\right)\right|\right)=\sup \left\{\left|T^{\prime}(y)\left(x_{n_{m}}\right)\right|,|y| \leq f_{m}\right\}$, for $m$ sufficiently large there exists some $y_{m} \in F^{\prime}$ such that $\left|y_{m}\right| \leq f_{m}$ and $\left|T^{\prime}\left(y_{m}\right)\left(x_{n_{m}}\right)\right|>\frac{\epsilon}{2}$. It is clear that $\left(y_{m}^{+}\right)$and $\left(y_{m}^{-}\right)$ are norm bounded disjoint sequences in $A^{+}$and so, by our hypothesis we obtain

$$
\begin{aligned}
\frac{\epsilon}{2} & <\left|T^{\prime}\left(y_{m}\right)\left(x_{n_{m}}\right)\right| \\
& \leq\left|T^{\prime}\left(y_{m}^{+}\right)\left(x_{n_{m}}\right)\right|+\left|T^{\prime}\left(y_{m}^{-}\right)\left(x_{n_{m}}\right)\right| \\
& \leq \sup _{k \in N}\left|T^{\prime}\left(y_{k}^{+}\right)\left(x_{n_{m}}\right)\right|+\sup _{k \in N}\left|T^{\prime}\left(y_{k}^{-}\right)\left(x_{n_{m}}\right)\right| \rightarrow 0,
\end{aligned}
$$

as $m \rightarrow \infty$. This leads to a contradiction, and we are done.

As a consequence of Theorem 2.7, we obtain the following result.

Corollary 2.8. Let $T$ be an order bounded operator from a Banach lattice $E$ into another Banach lattice $F$, and let $A$ be a norm bounded solid subset of $F^{\prime}$. The following statements are equivalent:

(1) $T^{\prime}(A)$ is an almost L-Dunford-Pettis set in $E^{\prime}$.

(2) $f_{n}\left(T\left(x_{n}\right)\right) \rightarrow 0$ as $n \rightarrow \infty$, for every disjoint weakly null sequence $\left(x_{n}\right)$, which is a Dunford-Pettis set in $E^{+}$and for each disjoint sequence $\left(f_{n}\right)$ in $A^{+}$.

Next, we derive another consequence of Theorem 2.7.

Corollary 2.9. Let $E$ be a Banach lattice and let $A$ be a norm bounded solid subset of $E^{\prime}$. The following statements are equivalent:

(1) $A$ is an almost L-Dunford-Pettis set in $E^{\prime}$.

(2) $\left\{f_{n}, n \in N\right\}$ is an almost L-Dunford-Pettis set in $E^{\prime}$, for each disjoint sequence $\left(f_{n}\right) \subset A^{+}=A \cap\left(F^{\prime}\right)^{+}$.

\section{Almost L-Dunford-Pettis set, aDPcc operator and PDPrcP}

The following theorem gives a new characterization of order bounded aDPcc operator from a Banach lattice $E$ into another $F$ in term of almost L-Dunford-Pettis sets in $E^{\prime}$.

Theorem 3.1. For an order bounded operator $T$ from a Banach lattice $E$ into another $F$. The following statements are equivalent:

(1) $T$ is an aDPcc operator.

(2) $T^{\prime}\left(B_{F^{\prime}}\right)$ is an almost L-Dunford-Pettis set in $E^{\prime}$.

(3) $\left\{T^{\prime}\left(f_{n}\right), n \in N\right\}$ is an almost L-Dunford-Pettis set in $E^{\prime}$, for each disjoint sequence $\left(f_{n}\right) \subset B_{F^{\prime}}^{+}$

(4) $f_{n}\left(T\left(x_{n}\right)\right) \rightarrow 0$ as $n \rightarrow \infty$, for every disjoint weakly null sequence $\left(x_{n}\right)$, which is a Dunford-Pettis set in $E^{+}$and for each disjoint sequence $\left(f_{n}\right) \subset B_{F^{\prime}}^{+}$.

Proof. (1) $\Leftrightarrow(2)$ Let $\left(x_{n}\right)$ be a disjoint weakly null sequence, which is a Dunford-Pettis subset of $E^{\prime}$. Since

$$
\left\|T\left(x_{n}\right)\right\|=\sup _{f \in T^{\prime}\left(B_{F^{\prime}}\right)}\left|f\left(x_{n}\right)\right|,
$$

then, it is clear that $T$ is an aDPcc operator if and only if $T^{\prime}\left(B_{F^{\prime}}\right)$ is an almost L-DunfordPettis in $E^{\prime}$.

$(2) \Leftrightarrow(3)$ Follows from Theorem 2.7.

$(3) \Leftrightarrow(4)$ Follows from Proposition 2.3.

As a simple consequence of Theorem 3.1, we get a characterization of PDPrcP in Banach lattices. 
Corollary 3.2. Let $E$ be a Banach lattice. The following statements are equivalent:

(1) E has the PDPrcP.

(2) $B_{E^{\prime}}$ is an almost L-Dunford-Pettis set.

(3) $\left\{f_{n}, n \in N\right\}$ is an almost L-Dunford-Pettis set in $E^{\prime}$, for each disjoint sequence $\left(f_{n}\right) \subset B_{E^{\prime}}^{+}$.

(4) $f_{n}\left(x_{n}\right) \rightarrow 0$ as $n \rightarrow \infty$, for every disjoint weakly null sequence $\left(x_{n}\right)$, which is a Dunford-Pettis set in $E^{+}$and for each disjoint sequence $\left(f_{n}\right) \subset B_{E^{\prime}}^{+}$.

In the next result, we obtain a new characterization of PDPrcP in Banach lattices in term of almost L-Dunford-Pettis sets.

Theorem 3.3. A Banach lattice $E$ has the PDPrcP if and only if every bounded subset of $E^{\prime}$ is an almost L-Dunford-Pettis set.

Proof. For the "if" part, since $B_{E^{\prime}}$ is an almost L-Dunford-Pettis set, by Corollary 3.2 we conclude that $E$ has the PDPrcP.

For the "only if" part, assume by way of contradiction that there exists a bounded subset $A$, which is not an almost L-Dunford-Pettis set of $E^{\prime}$. Then, there exists a disjoint weakly null sequence $\left(x_{n}\right)$, which is a Dunford-Pettis set of $E$ such that $\sup _{f \in A}\left|f\left(x_{n}\right)\right|>\epsilon>0$ for some $\epsilon>0$ and each $n$. Hence, for every $n$ there exists some $f_{n}$ in $A$ such that $\left|f_{n}\left(x_{n}\right)\right|>\epsilon$.

On the other hand, since $\left(f_{n}\right) \subset A$, there exists some $K>0$ such that $\left\|f_{n}\right\|_{E^{\prime}} \leq K$ for all $n$. Thus,

$$
\left|f_{n}\left(x_{n}\right)\right| \leq K\left\|x_{n}\right\|
$$

for each $n$, so by our hypothesis, $\left|f_{n}\left(x_{n}\right)\right| \rightarrow 0$ as $n \rightarrow \infty$, which is impossible. This completes the proof.

Let us define the following.

Definition 3.4. Let $E$ be a Banach lattice, $E$ has the property $(a)$ if for every weakly null sequence $\left(x_{n}\right)$, which is a Dunford-Pettis set in $E$ we have $\left|x_{n}\right| \rightarrow 0$ for $\sigma\left(E, E^{\prime}\right)$ as $n \rightarrow \infty$.

Remark 3.5. Let $E$ be a Banach lattice. Note that $E$ is discrete with order continuous norm $\Rightarrow$ the lattice operations of $E$ are weakly sequentially continuous (see Proposition 2.5.23 of [5]) $\Rightarrow E$ has the property $(a)$.

We need to recall of the following characterization of aDPcc operators, which is established in Theorem 3.9 of [4].

Theorem 3.6. An operator $T$ from a Banach lattice $E$ into a Banach space $Y$ is aDPcc if and only if $\left\|T\left(x_{n}\right)\right\| \rightarrow 0$ as $n \rightarrow \infty$ for every weakly null sequence $\left(x_{n}\right)$, which is a Dunford-Pettis set in $E^{+}$.

In the following result, we establish a sufficient condition such that the class of aDPcc operators and the class of DPcc operators coincide.

Theorem 3.7. Let $E$ be a Banach lattice and $Y$ be a Banach space such that $E$ has the property $(a)$, then each aDPcc operator from $E$ into $Y$ is DPcc.

Proof. Let $T$ be an aDPcc operator from $E$ into $Y$. We prove that $T$ is DPcc, let $\left(x_{n}\right)$ be a weakly null sequence, which is a Dunford-Pettis set in $E$. Since $E$ has the property $(a)$ then $\left(x_{n}^{+}\right)$and $\left(x_{n}^{-}\right)$be weakly null sequences in $E^{+}$, and it is clear that are Dunford-Pettis sets. Now, it follows from Theorem 3.6 that $\left\|T\left(x_{n}^{+}\right)\right\| \rightarrow 0$ and $\left\|T\left(x_{n}^{-}\right)\right\| \rightarrow 0$ as $n \rightarrow \infty$. Thus,

$$
\left\|T\left(x_{n}\right)\right\|=\left\|T\left(x_{n}^{+}\right)-T\left(x_{n}^{-}\right)\right\| \leq\left\|T\left(x_{n}^{+}\right)\right\|+\left\|T\left(x_{n}^{-}\right)\right\| \rightarrow 0 \text { as } n \rightarrow \infty,
$$

and we are done. 
Now, from Theorem 3.7 and Corollary 3.20 of [4], we derive

Corollary 3.8. Let $E$ and $F$ be two Banach lattices such that $E$ has the property $(a)$ or $F$ is discrete with order continuous norm, then each positive aDPcc operator from $E$ into F is DPcc.

The following result give a necessary and sufficient condition such that each order interval in a topological dual Banach lattice is an L-Dunford-Pettis set.

Proposition 3.9. Let $E$ be a Banach lattice. The following statements are equivalent:

(1) For every $f \in\left(E^{\prime}\right)^{+},[-f, f]$ is an L-Dunford-Pettis set in $E^{\prime}$.

(2) E has the property (a).

Proof. Let $\left(x_{n}\right)$ be a weakly null sequence, which is a Dunford-Pettis set of $E$, then the result follows from the equality:

$$
f\left(\left|x_{n}\right|\right)=\sup \left\{\left|g\left(x_{n}\right)\right|: g \in[-f, f]\right\},
$$

for every $f \in\left(E^{\prime}\right)^{+}$and every $n$.

We need the following proposition.

Proposition 3.10. A Banach space $X$ has the DPrcP if and only if the closed unit ball $B_{X^{\prime}}$ of $X^{\prime}$ is L-Dunford-Pettis.

Proof. Let $\left(x_{n}\right)$ be a weakly null sequence, which is a Dunford-Pettis set of $X$, then the result follows from the equality:

$$
\left\|x_{n}\right\|=\sup _{f \in B_{X^{\prime}}}\left|f\left(x_{n}\right)\right|
$$

for every $n$.

Remark 3.11. It is clear that every L-Dunford-Pettis set in a dual Banach lattice is almost L-Dunford-Pettis, but the converse is not true in general. In fact, if we put $E=$ $L^{1}[0,1] \oplus L^{2}[0,1]$ then, $E$ has the PDPrcP but does not have the DPrcP (see Example 3.4 of [4]), hence from Corollary 3.2 and Proposition 3.10, we see that the closed unit ball $B_{E^{\prime}}$ is an almost L-Dunford-Pettis set but it is not L-Dunford-Pettis.

Now, we are in a position to give our major result, and we characterize Banach lattice $E$ such that each almost L-Dunford-Pettis set of $E^{\prime}$ is L-Dunford-Pettis.

Theorem 3.12. Let $E$ be a Banach lattice. The following statements are equivalent:

(1) Each almost L-Dunford-Pettis set of $E^{\prime}$ is L-Dunford-Pettis.

(2) E has the property (a).

(3) Each aDPcc operator from $E$ to any Banach lattice $F$ is DPcc.

(4) Each aDPcc operator from $E$ to $\ell^{\infty}$ is DPcc.

Proof. (1) $\Rightarrow(2)$ Let $f \in\left(E^{\prime}\right)^{+}$then, $[-f, f]$ is an almost L-Dunford-Pettis set in $E^{\prime}$ (see Proposition 2.4), and by our hypothesis, we have that $[-f, f]$ is an L-Dunford-Pettis set in $E^{\prime}$. Now, from Proposition 3.9, we see that $E$ has the propery $(a)$.

$(2) \Rightarrow(3)$ Let $T$ be an aDPcc operator from $E$ to any Banach lattice $F$, since $E$ has the property $(a)$ then, by Theorem 3.7, $T$ is DPcc operator.

$(3) \Rightarrow(4)$ Obvious.

$(4) \Rightarrow(1)$ Suppose by way of contradiction that there exist an almost L-Dunford-Pettis set $A$ in $E^{\prime}$ which is not L-Dunford-Pettis. As $A$ is not L-Dunford-Pettis subst of $E^{\prime}$, so there exists a weakly null sequence $\left(x_{n}\right)$, which is a Dunford-Pettis subset of $E$ such that $\sup _{f \in A}\left|f\left(x_{n}\right)\right|>\epsilon>0$ for some $\epsilon>0$ and each $n$. Hence, for every $n$ there exists some $f_{n}$ in $A$ such that $\left|f_{n}\left(x_{n}\right)\right|>\epsilon$.

On the other hand, consider the operator $T: E \rightarrow \ell^{\infty}$ defined by 


$$
T(x)=\left(f_{n}(x)\right)_{n=0}^{\infty} \text { for all } x \in E .
$$

We show that $T$ is aDPcc operator. Since $A$ is almost L-Dunford-Pettis subst of $E^{\prime}$, then for every disjoint weakly null sequence $\left(y_{m}\right)$, which is a Dunford-Pettis of $E$, we obtain

$$
\begin{aligned}
\left\|T\left(y_{m}\right)\right\|_{\infty} & =\left\|\left(f_{n}\left(y_{m}\right)\right)_{n=0}^{\infty}\right\|_{\infty} \\
& =\sup _{n \in N}\left|f_{n}\left(y_{m}\right)\right| \\
& \leq \sup _{f \in A}\left|f\left(y_{m}\right)\right| \rightarrow 0,
\end{aligned}
$$

as $m \rightarrow \infty$, this prove that $T$ is aDPcc, and by our hypothesis we see that $T$ is DPcc.

Now, we have

$$
\epsilon<\left|f_{n}\left(x_{n}\right)\right| \leq\left\|T\left(x_{n}\right)\right\|_{\infty} \rightarrow 0, \text { as } n \rightarrow \infty,
$$

which is impossible, and this ends the proof.

Consequently, we obtain some sufficient conditions such that the PDPrcP and DPrcP in Banach lattice conicide.

Corollary 3.13. Let $E$ be a Banach lattice. Suppose that one of the following assertions is valid:

(1) Each almost L-Dunford-Pettis set of $E^{\prime}$ is L-Dunford-Pettis.

(2) E has the property (a).

(3) The lattice operations of $E$ are weakly sequentially continuous.

(4) $E$ is discrete.

(5) Each aDPcc operator from $E$ to $\ell^{\infty}$ is DPcc.

Then, $E$ has the PDPrcP if and only if $E$ has the DPrcP.

Proof. (1), (2) and (5) Follows from Theorem 3.12, in particular, we put in assertion (3) of this Theorem $F=E$ and $T=I d_{E}: E \rightarrow E$ the identity operator.

(3) Follows from Remark 3.5 and (2).

(4) If $E$ has the PDPrcP, then, its norm is order continuous, and as $E$ is discrete so by Remark 3.5 and assertion (3), we deduce that $E$ has the DPrcP, and this completes the proof.

\section{4. aL-Dunford-Pettis property in Banach lattices}

Let $E$ be a Banach lattice, note that each relatively weakly compact subset $A$ of a dual topological Banach lattice $E^{\prime}$ is L-Dunford-Pettis (see Proposition 2.3 of [6]), and hence $A$ is almost L-Dunford-Pettis. The converse of this property is not true in general, in fact, the closed unit ball $B_{\ell \infty}$ of $\ell^{\infty}$ is almost L-Dunford-Pettis set (see Corollary 3.2), but it is not relatively weakly compact.

Now, we give the following definition.

Definition 4.1. A Banach lattice $E$ has the aL-Dunford-Pettis property, if every almost L-Dunford-Pettis set in $E^{\prime}$ is relatively weakly compact.

Note that an aDPcc operator is not weakly compact in general. In fact, $I d_{\ell^{1}}$ is aDPcc, but it is not weakly compact.

Used the idea of aL-Dunford-Pettis property in Banach lattice, we establish the weak compactness of aDPcc operators.

Theorem 4.2. Let $E$ be a Banach lattice, then, the following assertions are equivalent:

(1) E has the aL-Dunford-Pettis property,

(2) for each Banach space $Y$, every aDPcc operator from $E$ into $Y$ is weakly compact,

(3) every aDPcc operator from $E$ into $\ell^{\infty}$ is weakly compact. 
Proof. $(1) \Rightarrow(2)$ Suppose that $E$ has the aL-Dunford-Pettis property and $T: E \rightarrow Y$ is aDPcc operator. Thus $T^{\prime}\left(B_{Y^{\prime}}\right)$ is an almost L-Dunford-Pettis set in $E^{\prime}$. So by hypothesis, it is relatively weakly compact and $T$ is a weakly compact operator.

(2) $\Rightarrow(3)$ Obvious.

$(3) \Rightarrow(1)$ If $E$ does not have the aL-Dunford-Pettis property, there exists an almost L-Dunford-Pettis subset $A$ of $E^{\prime}$ which is not relatively weakly compact. So there is a sequence $\left(f_{n}\right) \subseteq A$ with no weakly convergent subsequence. Now, we show that the operator $T: E \rightarrow \ell^{\infty}$ defined by $T(x)=\left(f_{n}(x)\right)$ for all $x \in E$ is aDPcc but it is not weakly compact. As $\left(f_{n}\right) \subseteq A$ is almost L-Dunford-Pettis set, then for every disjoint weakly null sequence $\left(x_{m}\right)$, which is a Dunford-Pettis set in $E$ we have

$$
\left\|T\left(x_{m}\right)\right\|=\sup _{n}\left|f_{n}\left(x_{m}\right)\right| \rightarrow 0, \text { as } m \rightarrow \infty,
$$

so $T$ is aDPcc operator. Hence $T^{\prime}\left(\left(\lambda_{n}\right)_{n=1}^{\infty}\right)=\sum_{n=1}^{\infty} \lambda_{n} f_{n}$ for every $\left(\lambda_{n}\right)_{n=1}^{\infty} \in \ell^{1} \subset\left(\ell^{\infty}\right)^{\prime}$. If $e_{n}^{\prime}$ is the usual basis element in $\ell^{1}$ then $T^{\prime}\left(e_{n}^{\prime}\right)=f_{n}$, for all $n \in N$. Thus, $T^{\prime}$ is not a weakly compact operator and neither is $T$. This finishes the proof.

As a consequence of Theorem 4.2, we derive the following result.

Corollary 4.3. A PDPrc space has the aL-Dunford-Pettis property if and only if it is reflexive.

Proof. $(\Rightarrow)$ If a Banach lattice $E$ has the PDPrcP, then the identity operator $I d_{E}$ on $E$ is aDPcc. As $E$ has the aL-Dunford-Pettis property, it follows from Theorem 4.2 that $I d_{E}$ is weakly compact, and hence $E$ is reflexive.

$(\Leftarrow)$ Obvious.

Remark 4.4. Note that the Banach lattice $\ell^{1}$ is not reflexive and has the PDPrcP, then from Corollary 4.3, we conclude that $\ell^{1}$ does not have the aL-Dunford-Pettis property.

Acknowledgment. The author would like to thank the referee for his comments which have improved this paper.

\section{References}

[1] C.D. Aliprantis and O. Burkinshaw, Positive operators, Reprint of the 1985 original. Springer, Dordrecht, 2006.

[2] K.T. Andrews, Dunford-Pettis sets in the space of Bochner integrable functions, Math. Ann. 241, 35-41, 1979.

[3] K. Bouras, Almost Dunford-Pettis sets in Banach lattices, Rend. Circ. Mat. Palermo. 62, 227-236, 2013.

[4] K. El Fahri, N. Machrafi and M. Moussa, Banach Lattices with the Positive DunfordPettis Relatively Compact Property, Extracta Math. 30 (2), 161-179, 2015.

[5] P. Meyer-Nieberg, Banach lattices, Universitext, Springer-Verlag, Berlin, 1991.

[6] A. Retbi and B. El Wahbi, L-Dunford-Pettis property in Banach spaces, Methods Funct. Anal. Topology, accepted.

[7] Y. Wen and J. Chen, Characterizations of Banach Spaces With Relatively Compact Dunford-Pettis Sets, Advances in Mathematics (China), 45 (1), 122-132, 2016. 